\title{
Uma Experiência do Uso Do Hardware Livre Arduino no Ensino De Programação De Computadores
}

\author{
David Albuquerque, Vitor Bremgartner, Hillermann Lima, Nilmara Salgado \\ Campus Manaus Distrito Industrial - Instituto Federal de Educação, Ciência e \\ Tecnologia do Amazonas (IFAM) \\ Av. Governador Danilo Areosa, nº 1672 - 69075-351 - Manaus - AM - Brasil \\ david.albmon@hotmail.com, \\ \{vitorbref, hillermann, nilmara.salgado\}@ifam.edu.br
}

\begin{abstract}
In programming languages courses, the difficulty that many students have to learn this subject is notorious, because besides being abstract and conceptual, in the beginning, the course requires a lot of practice, and requires the student to think by a computational way in several solutions for problems using programming. The activities in the classroom and notebook or even on the computer become uninteresting because it does not allow the student view in the real world what was programmed. Therefore, the objective of this work is to introduce practices with free hardware Arduino in teaching programming language, because through this hardware we can create different situations that help learning the language commands, and also stimulate the computational thinking.
\end{abstract}

Resumo. Nos cursos de linguagens de programação, é notória a dificuldade de muitos alunos em aprender tal assunto, pois além de ser abstrato e conceitual, em princípio, a disciplina exige muita prática, e requer que o aluno pense de maneira computacional nas mais diversas soluções de problemas usando programação. As atividades em sala de aula e no caderno ou até no computador se tornam desinteressantes, pois não permitem que o mesmo visualize no mundo real o que foi programado. Por isso, o objetivo deste trabalho é introduzir práticas com o hardware livre Arduino no ensino de linguagem de programação, pois através do hardware podemos criar diversas situações que auxiliem no aprendizado dos comandos de linguagem, e também estimulem o pensamento computacional.

\section{Introdução}

Costumeiramente, no ensino técnico de áreas tecnológicas como Informática, Mecatrônica e afins, muitos alunos demonstram dificuldades de aprendizado nas disciplinas de linguagem de programação. São vários os fatores que causam essa situação, dentre os quais podemos citar: dificuldade de desenvolvimento do raciocínio lógico, falta de percepção sobre onde e como aplicar esses conhecimentos, desânimo causado por esses motivos e também por assumir que terá muita dificuldade em conseguir aprovação na disciplina [Ribeiro et al. 2011; Cambruzzi et al. 2015]. A grande quantidade de informações novas que os alunos precisam aprender logo nas primeiras etapas do curso são normalmente conceitos e abstrações, estudados apenas em sala de aula, no projetor de slides, com exemplos no caderno, e pouca experimentação prática, dificultando o entendimento e consequentemente, o desenvolvimento do raciocínio lógico [Ribeiro et al. 2011; Zanetti et al. 2015; Cambruzzi et al 2015]. 
No caso das áreas tecnológicas industriais, como Mecatrônica, Eletrônica e outras, o ensino da linguagem de programação muitas vezes parece distante da realidade que os alunos terão após se formarem [Ribeiro et al. 2011; Romero-Zaliz et al 2014]. As aulas práticas acontecem em laboratórios de Informática com alunos aprendendo a escrever programas que calculam as raízes de uma equação, ou a média de dois números, ou que gerenciam contas bancárias, quando na verdade poderiam estar aprendendo a programar um painel de $L E D s$, uma esteira, um braço robótico, ou seja, assuntos que seriam mais próximos da área de atuação do aluno.

Um outro fator causador desse problema é o modelo de ensino instrucionista, ainda usado em muitas instituições [Ribeiro et al. 2011]. O modelo instrucionista é aquele que tem o professor como protagonista, ou seja, apenas o professor transmite o conhecimento para o aluno que simplesmente copia as informações aprendidas, porém muitas vezes sem compreender, tornando a aula desinteressante, pois se torna uma repetição mecânica, dificultando a relação entre o conhecimento e o aluno. É diferente do construtivismo, onde o aluno é o personagem principal e o professor o mediador. $\mathrm{O}$ aluno constrói os seus próprios conhecimentos através da exploração e da descoberta. [Ribeiro et al. 2011; Cambruzzi et al. 2015]

Muitos estudos têm sido realizados para mudar essa situação e vários deles sugerem a Robótica Educacional (RE) como alternativa. Através de diversas pesquisas, a RE tem se mostrado muito útil para auxiliar alunos e professores no ensino de disciplinas de linguagens de programação e até outras disciplinas como Matemática, Física, etc. Para [Lessa et al. 2015], a robótica oferece "a possibilidade de materialização daquilo que, até então, era visível somente na tela do computador". Dessa forma, "na aula com RE o aluno pensa, manuseia, constrói, executa, vê o que dá certo, depura o que está errado e reexecuta, ou seja, é o esmiuçar da teoria através da prática." [Cambruzzi et al. 2015]. Conforme [Ribeiro et al. 2011], a RE é uma forma de implementar as práticas construtivistas, pois ela permite que o aluno alcance o aprendizado através da busca e investigação.

Assim, este trabalho pretende utilizar a RE como alternativa para o ensino de linguagem de programação, estimulando o Pensamento Computacional, em uma turma do Ensino Médio Integrado em Mecatrônica do Instituto Federal de Educação, Ciência e Tecnologia do Amazonas (IFAM) do Campus Manaus Distrito Industrial (CMDI). Para isso, foi escolhido o hardware livre Arduino [Arduino 2015], onde foi realizado um experimento em laboratório consistindo em uma aplicação real do cotidiano dos alunos, a fim de fundamentar melhor os conceitos de programação estruturada.

Além desta Introdução, este artigo está dividido da seguinte maneira: na seção 2 é apresentado o referencial teórico sobre o hardware Arduino. Na seção 3 introduz-se o conceito de Pensamento Computacional. Na seção 4 estão trabalhos relacionados. Na seção 5 serão mostrados os métodos utilizados neste trabalho, enfatizando o experimento realizado em laboratório de programação. Os resultados estão na seção 6, e há algumas considerações finais na seção 7, seguida pelas referências.

\section{Arduino}

Nesse contexto, o hardware Arduino foi escolhido pela variedade de experimentos que podem ser feitos e pela linguagem de programação, que é similar à linguagem C.

Como o próprio fabricante o descreve, 
V Congresso Brasileiro de Informática na Educação (CBIE 2016)

Anais do XXII Workshop de Informática na Escola (WIE 2016)

Arduino é uma plataforma de prototipagem de código aberto baseada em hardware e software fáceis de usar. As placas Arduino são capazes de ler sinais de entrada - luz em um sensor, dedo em um botão ou mensagem no Twitter - e transformá-la num sinal de saída - ativando um motor, ligando um LED, publicando alguma coisa online. [Arduino 2015].

A Figura 1 mostra uma placa Arduino Uno.

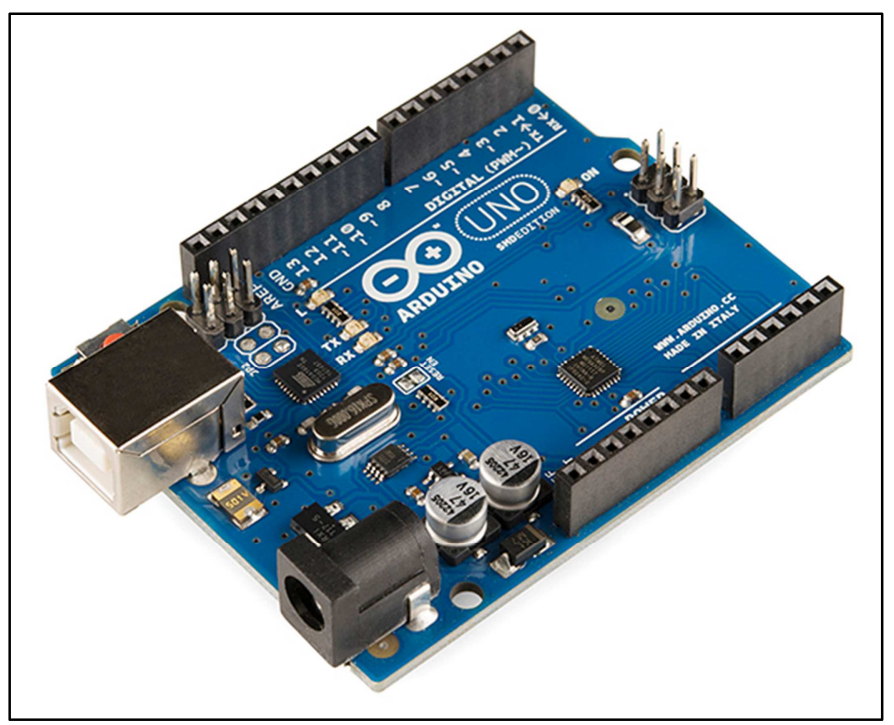

Figura 1 Arduino UNO.

Arduino surgiu no Ivrea Interaction Design Institute, na Itália, em 2005, como uma ferramenta para prototipagem rápida, objetivando estudantes que nunca estudaram programação ou eletrônica. Conforme o Arduino foi abrangendo mais pessoas, a placa começou a ser mudada para se adaptar a novas necessidades e desafios, transformandose de uma simples placa de 8-bits para produtos de IoT (Internet of Things), vestíveis, impressoras 3D e ambientes incorporados.

Atualmente, o Arduino possui uma grande popularidade, sendo utilizado em diversas aplicações, não somente na área eletrônica, mas até mesmo na área artística, educação, lazer, etc, e possui também várias comunidades de usuários, no mundo todo, compartilhando conhecimentos sobre o hardware [Alves et al. 2012].

\section{Pensamento Computacional}

Aprender linguagem de programação exige do estudante que ele pense na solução de um problema utilizando os recursos que aquela linguagem possui, ou seja, ele precisa pensar em como solucionar um problema escrevendo em código que possui palavras (funções) específicas. Essa forma de pensar é chamada Pensamento Computacional [Wing 2006] [Rodriguez et al. 2015].

$\mathrm{Na}$ verdade, esse conceito é muito mais abrangente, porém é preciso entender a diferença entre computação e informática antes. Informática é o ensino de aplicativos como editores de texto, editores de planilha, navegadores, e até algumas linguagens de programação. Enquanto que a computação é mais antiga e mais abrangente, ela existe desde quando o homem começou a criar máquinas de calcular. Ela é a ciência que 
V Congresso Brasileiro de Informática na Educação (CBIE 2016)

Anais do XXII Workshop de Informática na Escola (WIE 2016)

estuda a complexidade computacional, sistemas computacionais, sistemas operacionais, bancos de dados, redes, etc.

Portanto, o Pensamento Computacional não é apenas pensar em como solucionar problemas escrevendo programas, mas sim saber raciocinar sobre como usar os recursos de um computador, ou outra máquina computacional (neste caso, o Arduino) de forma a melhorar a capacidade cognitiva ou operacional do ser humano [França et al. 2014].

Sendo assim, a utilização do Arduino para o ensino de programação de computadores é uma das aplicações do Pensamento Computacional.

\section{Trabalhos Relacionados}

Há muitos trabalhos relacionados ao uso da Robótica em sala de aula para auxiliar no aprendizado de programação, tanto em turmas do Ensino Médio como do Superior, e há também uma variedade de ferramentas utilizadas. A seguir serão apresentados alguns deles.

No trabalho de [Ribeiro et al. 2011], os autores experimentaram a robótica no Ensino Superior para o ensino de linguagem de programação através do kit Mindstorms NXT 2.0, que é comercializado pela empresa LEGO, e a biblioteca LeJOS (Lego Java Operating System) [LEGO 2016]. O kit é composto de várias partes que se encaixam contendo sensores, atuadores e um controlador que recebe o programa. A biblioteca LeJOS é um projeto open-source com objetivo de permitir a programação dos robôs LEGO. O experimento foi feito em turmas do primeiro e segundo período dos cursos de computação e engenharia de produção em uma Universidade Federal no estado do Rio de Janeiro. Basicamente a ideia era fazer os alunos controlarem o movimento de um carrinho através da programação e ao final das aulas os alunos avaliaram a experiência que tiveram e foi constatado que a RE é uma forte estimuladora do aprendizado.

No trabalho de [Romero-Zaliz et al. 2014], a RE é utilizada, através do Arduino, para ensinar $\mathrm{C} / \mathrm{C}++$ e MATLAB em um curso introdutório de programação de nível superior. Foram desenvolvidos vários módulos de aprendizagem para aprimorar a metodologia tradicional de ensino. Os estudantes foram divididos em dois grupos: o grupo de controle foi submetido às metodologias tradicionais apenas, enquanto o grupo experimental teve sua metodologia aprimorada com o Arduino. Os resultados finais deste trabalho mostram que os alunos do grupo experimental acharam os módulos proveitosos e divertidos, e tiveram um melhor aprendizado do que os alunos do grupo de controle.

O ambiente Scratch [S4A 2016] para Arduino é utilizado como ferramenta no trabalho de [Zanetti et al. 2015], com o objetivo de desenvolver o pensamento computacional. Foi realizado primeiramente uma oficina e então uma prática com alunos com objetivo de avaliar o uso da RE. Os alunos eram do primeiro ano do Ensino Médio integrado ao técnico em Informática, ainda em processo de aprendizagem, porém já com dificuldades, e que nunca tiveram contato com RE. Porém, ao final das práticas, através de um questionário aplicado aos alunos, constatou-se que houveram melhorias no aprendizado, principalmente com relação a depuração de erros, e que a RE foi considerada, pelos alunos, um método mais interessante de aprender.

No trabalho de [Cambruzzi et al. 2015], através do uso de RE como objetos de aprendizagem (qualquer material que pode ser utilizado ou referenciado durante o 
processo de ensino-aprendizagem) foi também utilizado o kit da LEGO Mindstorms e também a ideia de separar os alunos em turmas diferentes: método tradicional (turma A) e RE (turma B), sendo aplicados testes (qualitativos e quantitativos) no início e no final do curso para verificar a eficácia da RE, nos quais percebeu-se que a turma B obteve melhores resultados que a turma A.

No artigo de [Melo et al. 2014], os autores propuseram um curso dividido em duas partes: a primeira consistia no ensino da programação e resolução de exercícios, e a segunda em aplicar os conhecimentos obtidos na primeira fase ao Arduino. Ao final de cada etapa foi aplicado um teste com os alunos. Os participantes eram estudantes do primeiro ano do Ensino Médio Integrado ao Técnico em Informática. O primeiro teste aplicado a turma revelou a grande dificuldade da turma no aprendizado, inclusive houveram muitas questões em branco, enquanto que o segundo teste mostrou resultados bem melhores que o primeiro.

Tendo observado os trabalhos relacionados, fica evidente que existem muitas abordagens na literatura a respeito do ensino de programação para estudantes de cursos de ciências exatas. $\mathrm{O}$ nosso trabalho apresenta um método de estímulo do Pensamento Computacional no ensino de linguagem de programação, principalmente para alunos iniciantes na área, algo que os faça verem de perto como os programas de computador funcionam. E por isso a plataforma Arduino torna-se bastante recomendável no caso desse trabalho, pois além de envolver conteúdos de programação também abrange os tópicos de eletrônica, que são muito importantes para os alunos do nível técnico que trabalham nessa área. Além disso, nossa abordagem apresenta-se de baixo custo, com a utilização do hardware Arduino, conforme será explicado na próxima seção.

\section{Métodos Empregados}

Foi realizado um experimento no IFAM/CMDI com alunos do segundo ano do curso Técnico em Mecatrônica, na faixa etária entre 16 e 17 anos (todos autorizados pelos pais ou responsável, por meio de um termo de consentimento). O objetivo do experimento era avaliar a aprendizagem dos alunos em conceitos de programação utilizando o hardware Arduino, a fim de que o mesmo pudesse aprimorar seus conhecimentos neste tema. Esta seção está dividida em duas etapas: planejamento e execução.

\subsection{Planejamento}

$\mathrm{Na}$ primeira etapa do projeto foi realizado o planejamento do protótipo, incluindo a perspectiva do projeto, tendo como atividades principais a construção física do ambiente a ser controlado, o algoritmo básico para implantação e a estimativa de componentes e materiais a serem utilizados no desenvolvimento. Durante o planejamento, também observamos a necessidade da turma passar por um treinamento inicial para utilização da ferramenta Arduino.

O cenário escolhido para esse experimento foi a instalação de uma lâmpada com interruptor para ligar e desligar a luz em um quarto. Para isso foi necessário colocar o interruptor de liga/desliga, o LED para representar a lâmpada assim como os demais itens para configuração do circuito que envolvam a parte de eletrônica. 
$\mathrm{Na}$ fase inicial desse projeto decidimos por utilizar um protótipo de baixa fidelidade em papel com a técnica de livro pop-up [eHow 2016]. Essa técnica possibilita que um livro se torne tridimensional, pois suas imagens 3D são formadas por dobraduras do papel que se transformam perfeitamente em algum cenário, personagem ou objeto da história narrada.

Esse recurso contribuiu para agilizarmos a elaboração do cenário, pois nessa fase já tínhamos as definições iniciais e ele poderia ser feito a mão utilizando materiais como papel tipo cartolina comum branco, tesouras, canetas coloridas e lápis. O resultado pode ser observado na Figura 2.

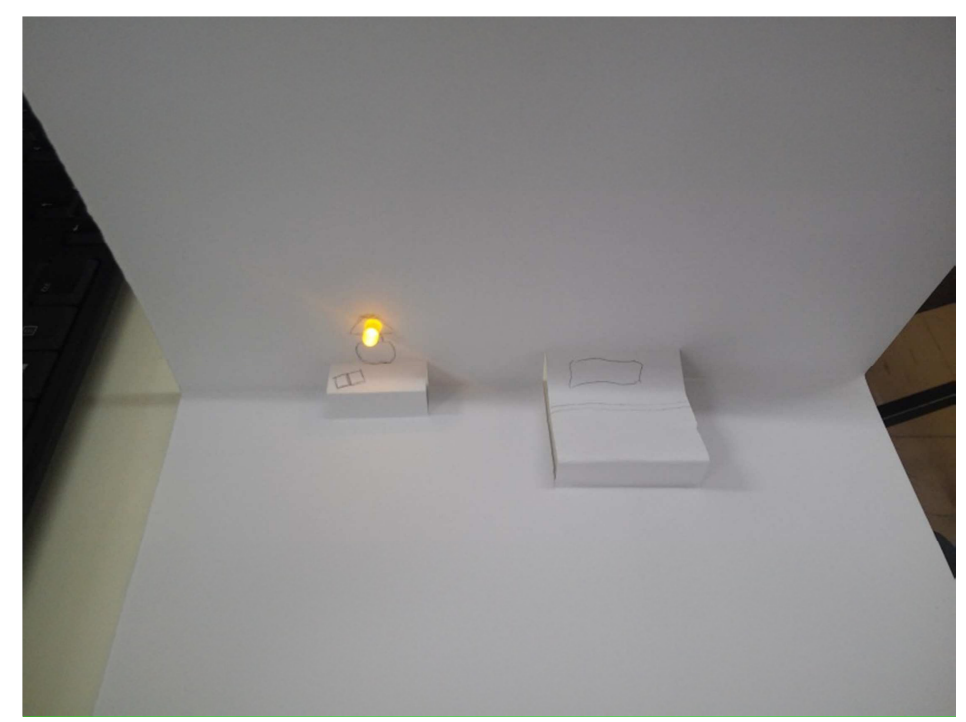

Figura 2 Protótipo em papel simulando layout de um quarto.

Para elaborar o circuito inicial que foi utilizado no protótipo em questão, foi obedecido o esquema elétrico mostrado na Figura 3 e para isso foram preparados os seguintes componentes:

- 1 breadboard;

- 1 arduino (modelo mega 2560);

- 1 LED (cores variadas);

- 1 resistência de 220 ohms;

- 6 jumpers para conexão;

- 1 chave liga/desliga.

O algoritmo elaborado deveria simular um cenário real de um ambiente físico. Para programação desse algoritmo foi utilizado o paradigma estruturado delimitando o mesmo na estrutura condicional Se-Senão, pois foi o conteúdo estudado na primeira etapa letiva da disciplina. 
V Congresso Brasileiro de Informática na Educação (CBIE 2016)

Anais do XXII Workshop de Informática na Escola (WIE 2016)

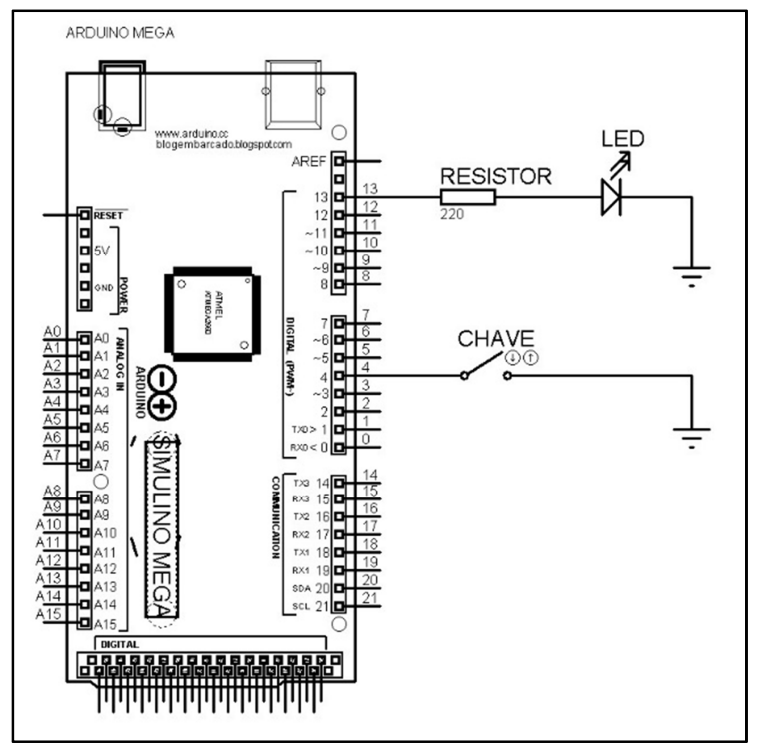

Figura 3 Esquema elétrico utilizado para montagem do circuito.

\subsection{Execução}

Na primeira aula prática, aconteceu o treinamento onde foi realizado um exercício com a ferramenta Arduino abordando sua teoria e a demonstração prática.

Posteriormente, na segunda aula prática, o experimento foi efetivamente aplicado. Para atingir as metas propostas, esse experimento seguiu o seguinte roteiro:

1. Apresentação do Experimento para os alunos.

2. Recebimento do Termo de Consentimento assinado pelos responsáveis.

3. Orientações sobre a prototipação em papel 3D.

4. Execução do experimento, em duas partes:

4.1 Elaboração do protótipo em papel.

4.2 Elaboração e montagem do circuito com o Arduíno.

$\mathrm{O}$ experimento foi realizado em duplas. Cada dupla de alunos recebeu um kit para montagem do circuito, o qual continha os materiais já citados na subseção 5.1.

O software utilizado para a elaboração do programa foi a própria ferramenta Arduino IDE (Integrated Development Environment). Ela já possui os recursos para utilização de suas bibliotecas, como também a verificação e o envio do código programado para a placa.

O código deveria fazer o Arduino ler uma informação do mundo real, por meio da chave, e com essa informação enviar um sinal para o mundo real, ou seja, comandar se o LED acende ou apaga. Observe na Figura 4 uma captura feita do quadro durante o trabalho. 
V Congresso Brasileiro de Informática na Educação (CBIE 2016)

Anais do XXII Workshop de Informática na Escola (WIE 2016)

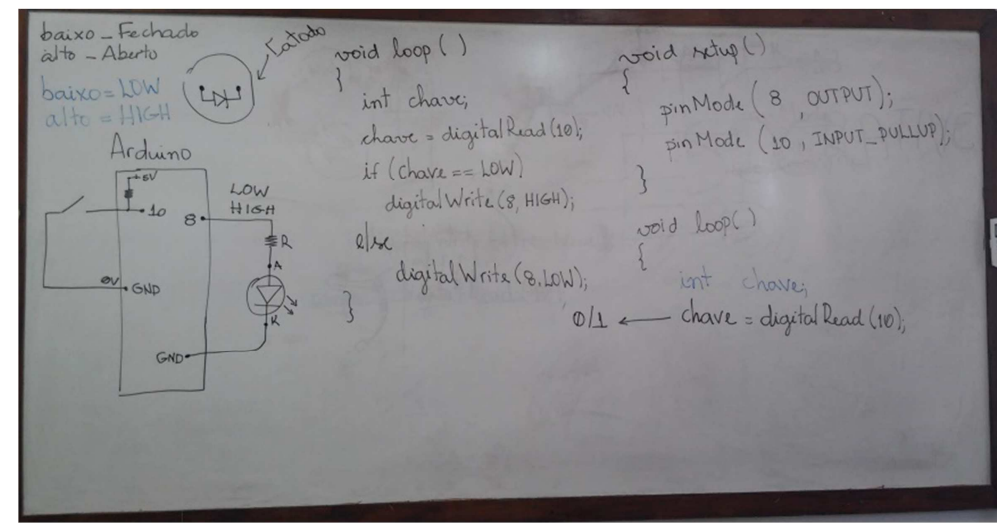

Figura 4 Captura do quadro com a proposta do código.

Para a execução do experimento no dia planejado foram utilizados três tempos de aula de 50 minutos cada. No primeiro tempo o trabalho teve inicio com uma reunião para orientação e esclarecimentos do experimento. Após esses passos, os alunos foram divididos em duplas para iniciar o trabalho do protótipo em 3D. Para isso foi exibido um vídeo e dado orientações sobre as vantagens de se trabalhar com prototipação em papel simulando cenários reais. Nos dois tempos seguintes foram trabalhados a montagem do circuito e o código. Durante a finalização do eperimento os alunos puderam avaliar a aula qualitativamente, por meio de uma entrevista.

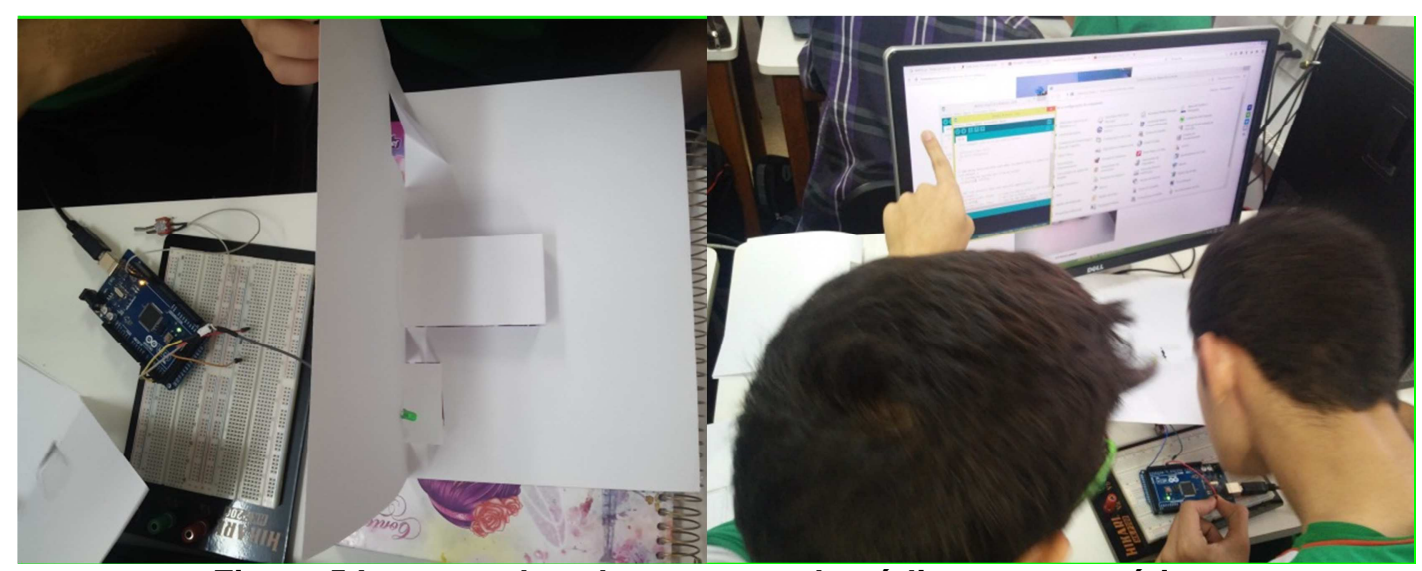

Figura 5 Imagens dos alunos testando código com protótipo.

\section{Análise dos Resultados}

Iniciamos a análise dos dados com a verificação do perfil dos 18 alunos que participaram do experimento. Sendo uma turma de faixa etária entre 16 e 17 anos, os alunos em questão estão cursando o segundo ano do ensino técnico. Essa modalidade abrange no primeiro e segundo ano disciplinas técnicas, como: Desenho Técnico, Eletricidade, Eletrônica Analógica I, Eletrônica Digital, Equipamentos de Automação e Controle, além de Algoritmos e Programação, que utiliza a linguagem C.

A partir do cenário proposto observamos que no momento de associar a estrutura já estudada ao experimento, os alunos não tiveram dificuldades e concluíram o código sem maiores dificuldades.

Os alunos responderam alguns questionamentos para avaliar a produtividade da aula. As respostas foram bastante positivas e interessantes. Ao serem perguntados se já 
V Congresso Brasileiro de Informática na Educação (CBIE 2016)

Anais do XXII Workshop de Informática na Escola (WIE 2016)

tinham alguma experiência anterior com Arduino somente dois alunos responderam que sim; um deles disse ter comprado um kit iniciante para fazer experiências em casa, ao saber que teria aulas com Arduino.

Quando perguntados se gostaram da aula todos responderam sim ou muito. Todos concordaram que a aula foi mais interessante do que as aulas tradicionais (apenas exposição em datashow e códigos-fonte nos computadores do laboratório), fato ressaltado pela professora, a qual notou que todos estavam realmente em silêncio e prestando atenção na aula.

Ao serem perguntados por que acharam a aula mais interessante as repostas foram variadas, onde aqui destacamos as principais: "na sala de aula a gente aprende $o$ conteúdo e aqui a gente coloca na prática e realmente entende" disse uma aluna. Um outro aluno fez um comentário sobre o prompt desinteressante que ele costuma ver nas aulas de linguagem C: "A aplicação na prática é bem mais divertida porque estamos aprendendo $C$ e só aquele prompt preto não é tão interessante e o Arduino é mais real." Uma outra aluna defende que as aulas em sala, no laboratório com o software Dev $\mathrm{C}++$, e com Arduino se completam. Ela diz: "em sala de aula é muito metódico, na sala de aula é somente conteúdo, é chato, dá sono, a gente não entende, aí quando a gente vai praticando a gente consegue assimilar o que a gente tá fazendo [...] no Dev C++ eu vou aprendendo a estrutura em si, no Arduino eu já vou praticando aquela estrutura que eu já tinha aprendido pra ver o que vai acontecer".

Todos os alunos alegaram não sentir grandes dificuldades, mas de fato foram notados pequenos empecilhos, por exemplo: alguns alunos não notaram que a IDE do arduino faz diferença entre letras maiúsculas e minúsculas, mas logo foram instruídos para não errarem o código; um outro aluno disse que a única dificuldade foi pelo fato da interface estar em inglês. É importante ressaltar também que um aluno demonstrou tanto interesse sobre Arduino que quis saber se seria possível, e como, controlar o acendimento das lâmpadas de sua casa com o Arduino utilizando um celular.

Percebe-se que o método proposto nesta aula prática de linguagem de programação utilizando o Arduino constitui num importante fator de estímulo de Pensamento Computacional dos alunos, uma vez que foi utilizado um cenário de exemplo da vida real para o aprendizado de estruturas condicionais.

\section{Considerações Finais}

A atividade realizada com os alunos e apresentada nesse trabalho é um exemplo de como é importante estimular o Pensamento Computacional, especificamente, o aprendizado de linguagem de programação em cursos técnicos, pois de fato muitos jovens que ingressam no Ensino Médio, ou que já o concluíram, buscam a qualificação em uma área técnica, mas sem estarem totalmente decididos sobre qual carreira seguirão. Assim, ao encararem pela primeira vez a disciplina de Algoritmos e Programação, normalmente sentem dificuldades. Logo, ficam desanimados a continuar, pensando até em mudar de área, visto que disciplinas de linguagem de programação abrange muitos cursos das áreas tecnológicas.

É importante que os alunos percebam que a programação não é algo entediante e impossível de aprender, mas sim que ela é uma ferramenta interessante e útil que está presente no cotidiano, não só em computadores, mas nos celulares, televisores, caixas eletrônicos, máquinas de lavar, máquinas fotográficas, aparelhos DVD, entre outros, ou seja, há várias possibilidades além da tela preta do prompt de comando. 
V Congresso Brasileiro de Informática na Educação (CBIE 2016)

Anais do XXII Workshop de Informática na Escola (WIE 2016)

Dessa forma, espera-se que o relato dos experimentos realizados nesse trabalho também sirvam de incentivo para que mais professores possam explorar novos métodos de ensino, seja em sala de aula, seja no laboratório, uma vez que neste trabalho a receptividade dos estudantes foi alta. Como trabalhos futuros, sugere-se a exploração de mais cenários onde possamos explorar outras estruturas dos algoritmos, como estruturas de repetição e estruturas de dados, além de explorar possibilidades de utilizar Arduino integrado a aplicações de dispositivos móveis.

\section{Agradecimentos}

Este trabalho recebeu o apoio do Programa de Apoio a Iniciação Científica (PAIC) da Fundação de Amparo e Pesquisa do Estado do Amazonas (FAPEAM).

\section{Referências}

Alves, R. M.; Silva, A.L.C.; Pinto, M.C.; Sampaio, F. F.; Elia, M. F.; (2012). Uso do hardware livre arduino em ambientes de ensino-aprendizagem. Jornada de Atualização em Informática na Educação - JAIE.

Arduino. (2015) https://www.arduino.cc/en/Guide/Introduction

Cambruzzi, E.; Souza. R. M.; (2015). Robótica Educativa na aprendizagem de Lógica de Programação: Aplicação e análise. Anais do XXI Workshop de Informática na Escola (WIE).

eHow. Passos para fazer um livro pop-up complexo (2016). http://www.ehow.com.br/passos-livro-popup-complexo-estrategia_15348/

França, R. S.; V. A. S. Ferreira V. A. S.; Almeida, L. C. F.; Amaral, H. J. C.; (2014). A disseminação do pensamento computacional na educação básica: lições aprendidas com experiências de licenciandos em computação. XXXIV Congresso da Sociedade Brasileira de Computação - CSBC.

LEGO Mindstorms. Home. (2016) http://www.lego.com/enus/mindstorms/?domainredir=mindstorms.lego.com

Lessa, V. E.; Forigo, F. M.; Teixeira A. C.; Licks, G. P. (2015). Programação de Computadores e Robótica Educativa na Escola: tendências evidenciadas nas produções do Workshop de Informática na Escola. Anais do XXI Workshop de Informática na Escola (WIE).

Melo, R. M.; Feliciano, F. D. O.; Brito, J. A.; Melo Filho, I. J.; Carvalho, R. S.; Gomes, A. S.; (2014) .Utilização do Arduino como Estratégia Pedagógica na Aprendizagem da Lógica de Programação.

Ribeiro, P. C.; Martins, C. B.; Bernardini, F.C.; (2011). A Robótica como Ferramenta de Apoio ao Ensino de Disciplinas de Programação em Cursos de Computação e Engenharia. XXII SBIE - XVII WIE. Aracaju.

Rodriguez, C. L.; Zem-Lopes, A. M.; Marques, L.; Isotani, S.; (2015). Pensamento Computacional: transformando ideias em jogos digitais usando o Scratch. Anais do XXI Workshop de Informática na Escola (WIE 2015).

Romero-Zaliz R.; Rubio, M. A.; Madrid, C. M. A. P.; (2014). Enhancing an introductory programming course with physical computing modules. Universidade de Granada. Espanha.

S4A. Scratch for Arduino (2016). http://s4a.cat/

Wing, J. Computational Thinking. Communications of the ACM, 3 ed.:33-35, March, 2006.

Zanetti, H. A. P.; Oliveira, C. L. V.; (2015). Prática de ensino de Programação de Computadores com Robótica Pedagógica e aplicação de Pensamento Computacional. Anais dos Workshops do IV Congresso Brasileiro de Informática na Educação (CBIE). 